\title{
Erratum to: Comparative analysis of germ cells and DNA of the genus Amblyomma: adding new data on Amblyomma maculatum and Amblyomma ovale species (Acari: Ixodidae)
}

\author{
Fredy Arvey Rivera-Páez ${ }^{1,2}$ - Bruno Rodrigues Sampieri ${ }^{3}$ - Marcelo Bahia Labruna ${ }^{4}$. \\ Renata da Silva Matos $^{1} \cdot$ Thiago Fernandes Martins $^{4} \cdot$ Maria Izabel Camargo-Mathias ${ }^{1}$
}

Published online: 20 September 2017

(C) Springer-Verlag GmbH Germany 2017

Erratum to: Parasitology Research

https://doi.org/10.1007/s00436-017-5592-x

The data in Table 3 were misaligned in the original version of this article. Correct table alignment is presented here.

The original article has been corrected.

The online version of the original article can be found at https://doi.org/ $10.1007 / \mathrm{s} 00436-017-5592-\mathrm{x}$

\footnotetext{
Maria Izabel Camargo-Mathias

micm@rc.unesp.br

1 Departamento de Biologia, Instituto de Biociências, UNESP -

Universidade Estadual Paulista, Avenida 24-A, 1515, Bairro Bela

Vista, Rio Claro, SP CEP13506-900, Brazil

2 Grupo de Investigación GEBIOME, Departamento de Ciencias Biológicas, Facultad de Ciencias Exactas y Naturales, Universidad de Caldas, Calle 65 No. 26-10 Apartado Aéreo 275 Manizales, Caldas, Colombia

3 Universidade Estadual de Campinas, Museu de Zoologia, R. Charles Darwin s/n, cidade universitária, Campinas, SP, Brazil

4 Departamento de Medicina Veterinária Preventiva e Saúde Animal, Faculdade de Medicina Veterinária e Zootecnia, Universsidade de São Paulo-USP, Av. Prof. Orlando Marques de Paiva, 87, CEP 05508-000, Cidade Universitária, São Paulo, SP, Brazil
} 
Table 3 Morphological characters matrix used for evaluation of the male reproductive system of ticks

\section{Character}

\begin{tabular}{|c|c|c|c|c|c|c|c|c|c|c|c|}
\hline \multirow{9}{*}{ 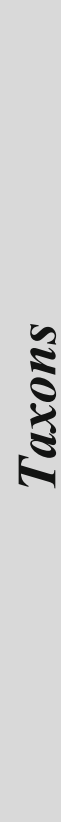 } & & 1 & 2 & 3 & 4 & 5 & 6 & 7 & 8 & 9 & 10 \\
\hline & A & 1 & 1 & 0 & 1 & 1 & 1 & 1 & 0 & 0 & 1 \\
\hline & B & 1 & 1 & 1 & 1 & 1 & 1 & 1 & 1 & 0 & 1 \\
\hline & C & 1 & 1 & 0 & 1 & 1 & 1 & 1 & 0 & 1 & 0 \\
\hline & D & 1 & 1 & 0 & 1 & 0 & 1 & 1 & 0 & 1 & 0 \\
\hline & $\mathbf{E}$ & 1 & 1 & 0 & -- & -- & -- & 1 & 1 & 1 & 0 \\
\hline & GE1 & 1 & 1 & 0 & 1 & 1 & 1 & 0 & 1 & 1 & 1 \\
\hline & GE2 & 0 & 0 & 0 & 0 & 0 & 0 & 0 & 0 & 0 & 0 \\
\hline & & ABCDE & ABCDE & ACDE & ABCD & $\mathrm{ABC}$ & ABCD & ABCDE & BE & CDE & $\mathbf{A B}$ \\
\hline
\end{tabular}

GE Grupo Externo, A A. aureolatum, B A. triste, $\boldsymbol{C}$ A. sculptum, D A. ovale, $\boldsymbol{E}$ A. maculatum 